\title{
ON PERTURBATIONS OF THE ISOMETRIC SEMIGROUP OF SHIFTS ON THE SEMIAXIS
}

\author{
G. G. AMOSOV, A. D. BARANOV, AND V. V. KAPUSTIN
}

\begin{abstract}
Perturbations $\left(\widetilde{\tau}_{t}\right)_{t \geq 0}$ of the semigroup of shifts $\left(\tau_{t}\right)_{t \geq 0}$ on $L^{2}\left(\mathbb{R}_{+}\right)$are studied under the assumption that $\widetilde{\tau}_{t}-\tau_{t}$ belongs to a certain Schatten-von Neumann class $\mathfrak{S}_{p}$ with $p \geq 1$. It is shown that, for the unitary component in the WoldKolmogorov decomposition of the cogenerator of the semigroup $\left(\widetilde{\tau}_{t}\right)_{t \geq 0}$, any singular spectral type may be achieved by $\mathfrak{S}_{1}$-perturbations. An explicit construction is provided for a perturbation with a given spectral type, based on the theory of model spaces of the Hardy space $H^{2}$. Also, it is shown that an arbitrary prescribed spectral type may be obtained for the unitary component of the perturbed semigroup by a perturbation of class $\mathfrak{S}_{p}$ with $p>1$.
\end{abstract}

\section{$\S 1$. INTRODUCTION}

Consider the isometric semigroup $\left(\tau_{t}\right)_{t \geq 0}$ of shifts on the space $L^{2}\left(\mathbb{R}_{+}\right)$,

$$
\left(\tau_{t} f\right)(x)=\left\{\begin{array}{ll}
f(x-t), & x \geq t, \\
0, & x<t,
\end{array} \quad f \in L^{2}\left(\mathbb{R}_{+}\right) .\right.
$$

In this paper we are concerned with perturbations $\left(\widetilde{\tau}_{t}\right)$ of the semigroup $\left(\tau_{t}\right)$ satisfying the following properties:

$\left(\widetilde{\tau}_{t}\right)_{t \geq 0}$ is a strongly continuous semigroup of isometric operators on $L^{2}\left(\mathbb{R}_{+}\right)$;

the difference $\widetilde{\tau}_{t}-\tau_{t}$ belongs to a certain Schatten-von Neumann ideal $\mathfrak{S}_{p}$ for every $t>0$.

The central problem considered in this paper is to describe all possible spectral types of perturbed isometric semigroups. The spectral type of a semigroup determines the semigroup uniquely up to unitary equivalence; it is defined by the spectral type of the cogenerator of the group (the definition can be found in $\S 2$ ). For $p=1$, the stability of the absolutely continuous spectrum of the unitary dilation implies that the absolutely continuous parts of the cogenerators for the unitary dilations of the semigroups $\left(\tau_{t}\right)$ and $\left(\widetilde{\tau}_{t}\right)$ are unitarily equivalent (see $\S 2$ for the details). The cogenerator of the semigroup $\left(\tau_{t}\right)$ is unitarily equivalent to the unilateral shift operator on the Hardy space $H^{2}$. Thus, for $p=1$, our problem reduces to the description of all possible singular parts, and we show that any singular type may be realized by some semigroup $\left(\widetilde{\tau}_{t}\right)_{t \geq 0}$. For $p=2$, in [1, 2] it was shown that any spectral type of the unitary component may occur; for the case of a singular spectral measure, a model of such perturbations was constructed. Here we show that similar results are valid for all $p>1$.

2010 Mathematics Subject Classification. Primary 47D03, 47B37, 47B10.

Key words and phrases. Semigroup of shifts, trace-class perturbation, Schatten-von Neumann ideals, Hardy space, inner function.

Partially supported by the Federal Program 2.1.1/1662, by RFBR (grant no. 08-01-00723), and by the President of Russian Federation grant no. NSH 2409.2008.1. 
A possible motivation for our study is the relationship with Markov perturbations of the unitary group of shifts. The semigroup $\left(\tau_{t}\right)_{t \geq 0}$ is the restriction to $L^{2}\left(\mathbb{R}_{+}\right)$of the unitary group of shifts $\left(\gamma_{t}\right)_{t \in \mathbb{R}},\left(\gamma_{t} f\right)(x)=f(x-\bar{t})$, on the space $L^{2}(\mathbb{R})$ on the line (we identify the spaces $L^{2}\left(\mathbb{R}_{+}\right)$and $L^{2}\left(\mathbb{R}_{-}\right)$with the subspaces of functions of class $L^{2}(\mathbb{R})$ identically equal to zero on the semiaxes $\mathbb{R}_{-}$and $\mathbb{R}_{+}$, respectively). Consider a perturbed unitary group $\left(\widetilde{\gamma}_{t}\right)_{t \in \mathbb{R}}$ and assume that it has the so-called Markov property, which means that the perturbed operators coincide with the unperturbed ones on the left semiaxis $\mathbb{R}_{-}$ for $t<0$, i.e.,

$$
\gamma_{t} f=\widetilde{\gamma}_{t} f \quad \text { if } \quad f=0 \text { on } \mathbb{R}_{+}, \quad t<0 .
$$

As usual, the Markov property may be interpreted in the sense that "the past does not depend on the future". Markov perturbations with the additional property $\widetilde{\gamma}_{t}-\gamma_{t} \in \mathfrak{S}_{2}$, $t \in \mathbb{R}$, were investigated by the first author (see, e.g., [15]) in connection with cocycle perturbations of the flow of Powers shifts [17.

Sometimes it will be convenient to work with the model in the Hardy space $H^{2}$ on the unit circle $\mathbb{T}$, which is unitarily equivalent to the original model (see $\S 4$ ). The semigroup $\left(\tau_{t}\right)_{t>0}$ on $L^{2}\left(\mathbb{R}_{+}\right)$is unitarily equivalent to the semigroup of operators on $H^{2}$ of multiplication by the functions $\phi_{t}, t \geq 0$, where

$$
\phi_{t}(z)=\exp \left(t \frac{z+1}{z-1}\right) .
$$

In this case the cogenerator of the unperturbed semigroup is the unilateral shift $S$, i.e., the operator of multiplication by $z$ in $H^{2}$. Denote by $\widetilde{S}$ the cogenerator of the perturbed semigroup; then the elements of this semigroup are of the form $\phi_{t}(\widetilde{S})$.

Now we introduce a new parameter of our model of perturbations, namely, an inner function $\theta$ in the unit disk (a function $\theta \in H^{2}$ is said to be inner if $|\theta|=1$ almost everywhere on $\mathbb{T}$ ). Consider the $S$-coinvariant subspace

$$
K_{\theta}=H^{2} \ominus \theta H^{2} .
$$

For the theory of the backward shift invariant subspaces, also called model subspaces, see [6, 21].

In what follows we are interested in perturbations $\widetilde{S}$ of the shift operator $S$ with the following properties:

(i) $\widetilde{S}$ is an operator on $H^{2}$, diagonal with respect to the decomposition $H^{2}=K_{\theta} \oplus \theta H^{2}$;

(ii) $\widetilde{S}$ acts on $\theta H^{2}$ as multiplication by $z$;

(iii) the restriction of $\widetilde{S}$ to $K_{\theta}$ is a unitary operator, and 1 is not its eigenvalue.

Conditions (i)-(iii) mean that $\widetilde{S}$ is an isometry, whose unitary and completely nonunitary parts act on $K_{\theta}$ and $\theta H^{2}$, respectively. The number 1 does not belong to the point spectrum of $\widetilde{S}$, and the isometric semigroup with cogenerator $\widetilde{S}$ is well defined.

Now we state our main results. First of them shows that any singular summand $V$ can be obtained by means of trace class perturbations of the isometric semigroup of shifts in the model on the unit circle.

Theorem 1.1. Let $V$ be a singular unitary operator of multiplicity $n \leq \infty$ such that 1 is not an eigenvalue of $V$, and let $\varepsilon>0$. Then there exist an inner function $\theta$ and an operator $\widetilde{S}$ with properties (i)-(iii) such that

a) the restriction of $\widetilde{S}$ to $K_{\theta}$ is unitarily equivalent to $V$;

b) $\operatorname{rank}(\widetilde{S}-S) \leq n$;

c) $\|\widetilde{S}-S\|_{\mathfrak{S}_{1}} \leq \varepsilon$;

d) $\phi_{t}(\widetilde{S})-\phi_{t}(S) \in \mathfrak{S}_{1}$ for all $t>0$. 
As an immediate consequence of Theorem 1.1, we obtain the following statement about perturbations of the semigroup of shifts on the semiaxis.

Theorem 1.2. Let $\left(\tau_{t}\right)_{t \geq 0}$ be the semigroup of shifts on $L^{2}\left(\mathbb{R}_{+}\right)$.

1) If $\left(\widetilde{\tau}_{t}\right)$ is an isometric semigroup such that $\widetilde{\tau}_{t}-\tau_{t} \in \mathfrak{S}_{1}$ for all $t \geq 0$, then the cogenerator of the semigroup $\left(\widetilde{\tau}_{t}\right)$ is unitarily equivalent to the direct sum of the shift operator (of multiplicity one) and a unitary operator with a singular spectral measure.

2) For any singular unitary operator $V$ with the only restriction that 1 does not belong to its point spectrum, there exists an isometric semigroup $\left(\widetilde{\tau}_{t}\right)$ whose cogenerator is unitarily equivalent to the operator $S \oplus V$, and $\widetilde{\tau}_{t}-\tau_{t} \in \mathfrak{S}_{1}$ for all $t \geq 0$.

As another corollary to Theorem 1.1 we show that a similar statement is true for $p>1$ without the assumption that the unitary operator $V$ is singular.

Theorem 1.3. Let $V$ be a unitary operator such that 1 is not an eigenvalue of $V$. Then there exist an inner function $\theta$ and an operator $\widetilde{S}$ satisfying (i)-(iii) for which the restriction of $\widetilde{S}$ to $K_{\theta}$ is unitarily equivalent to $V$ and $\phi_{t}(\widetilde{S})-\phi_{t}(S) \in \mathfrak{S}_{p}$ for all $p>1$.

For $p=2$, analogs of Theorems 1.1 and 1.3 were obtained in [1, 2]. Here we also consider separately the results concerning the class $\mathfrak{S}_{2}$, because in terms of our model their proofs simplify essentially and the conditions obtained may be expected to be sharp. The new results of this paper are related to narrower classes, i.e., $\mathfrak{S}_{p}$ with $p<2$, and especially with $p=1$.

In 16, 1], the construction of the operator $\widetilde{S}$ was based on the triangulation studied by Ahern and Clark [14. Here we use Clark's construction [18 instead, which establishes an isometric identification of the space $K_{\theta}$ with a space $L^{2}(\mu)$ for a certain singular measure $\mu$ on the unit circle. This approach allows us to relate approximation properties of the difference $\phi_{t}(\widetilde{S})-\phi_{t}(S)$ to differential properties of $\mu$.

The situation becomes essentially different when we pass to the "natural" unitary dilation of the perturbed semigroup $\widetilde{S}$ with properties (i)-(iii) (see $\S 2$ ). Let $U$ be the bilateral shift on $L^{2}(\mathbb{T})$, and let $\widetilde{U}$ be a dilation of $\widetilde{S}$ with the Markov property, i.e., such that $\widetilde{U}^{*}$ coincides with $U^{*}$ on $H_{-}^{2}$. It turns out that then $\phi_{t}(\tilde{U})-\phi_{t}(U)$ will never belong to $\mathfrak{S}_{1}$. However, for any unitary operator $V$ there exist a construction of $\widetilde{S}$ satisfying (i)-(iii) and a Markov unitary dilation $\widetilde{U}$ of $\widetilde{S}$ such that the restriction of $\widetilde{S}$ to $K_{\theta}$ is unitarily equivalent to $V$ and $\phi_{t}(\widetilde{U})-\phi_{t}(U) \in \mathfrak{S}_{p}$ for all $p>1$. Unitary dilations will be considered in detail elsewhere.

The paper is organized as follows. In $\S 2$ it is shown that the conditions imposed on the operator $V$ in the main results are necessary. After that, we first consider the case where $V$ is a singular unitary operator of multiplicity 1 and we may apply Clark's construction. The model is determined by an inner function $\theta$, or by a singular measure $\mu$ on the unit circle related to $\theta$ by formula (4) below, or by a measure $\nu$ on the real line associated with $\mu$. In this case we have $\operatorname{rank}(\widetilde{S}-S)=1$. We obtain conditions on $\mu$ and $\nu$ under which the operators $\phi_{t}(\widetilde{S})-\phi_{t}(S)$ belong to $\mathfrak{S}_{2}$ and $\mathfrak{S}_{1}$ for all $t>0$. We prove Theorem 1.1 in the special case of multiplicity 1 with the help of properties of the measures $\mu$ and $\nu$ from our construction, and then we use this special case to deduce the general case of the theorem. Theorems 1.2 and 1.3 are obtained as corollaries to Theorem 1.1 .

The authors are grateful to R. V. Romanov for useful discussions and for help with the proof of Theorem 2.1 and also to A. B. Aleksandrov for many helpful suggestions that have improved the exposition. 


\section{§2. UNITARY GROUPS, ISOMETRIC SEMIGROUPS, AND THEIR COGENERATORS}

In this section we recall basic properties of generators and cogenerators of strongly continuous unitary groups and their analogs for isometric semigroups. A detailed exposition of these topics can be found in [4, 10, 11.

If $\left(U_{t}\right)_{t \in \mathbb{R}}$ is a strongly continuous unitary group, then its generator $A$ is defined by $A x=\lim _{t \rightarrow 0} \frac{U_{t}-I}{t} x$ on the set of vectors $x$ for which the limit exists. Then $i A$ is a selfadjoint operator, and $U_{t}=\exp (t A)$. The cogenerator is the unitary operator $B=(A+I)(A-I)^{-1}$. A necessary and sufficient condition for a unitary operator to be a cogenerator of some unitary group is that its point spectrum does not contain the point 1. For the elements of the group we have the formula expressing them via the cogenerator:

$$
U_{t}=\phi_{t}(B)
$$

with $\phi_{t}$ defined by (2). Moreover, since

$$
\int_{0}^{+\infty} e^{-t} \phi_{t}(z) d t=\frac{1-z}{2}
$$

we obtain

$$
\int_{0}^{+\infty} e^{-t} U_{t} d t=\int_{0}^{+\infty} e^{-t} \phi_{t}(B) d t=\frac{I-B}{2}
$$

hence, the cogenerator can be expressed in terms of the elements of the group with $t \geq 0$ as

$$
B=I-2 \int_{0}^{+\infty} e^{-t} U_{t} d t
$$

Now, suppose that we are given a strongly continuous semigroup $\left(V_{t}\right)_{t \geq 0}$ of isometric operators on a Hilbert space $H_{0}$. Then we can consider its unitary dilation, i.e., a unitary group $\left(U_{t}\right)_{t \in \mathbb{R}}$ acting on a wider space $H$ such that $H_{0}$ is an invariant subspace for $U_{t}$ with $t>0$, and $V_{t}=\left.U_{t}\right|_{H_{0}}, t>0$ (see [10]). If $t \geq 0$, then $\phi_{t} \in H^{\infty}$. Therefore, $H_{0}$ is also an invariant subspace for the cogenerator $B$ of $\left(U_{t}\right)$, and it is natural to define the cogenerator of the isometric semigroup $\left(V_{t}\right)$ as the restriction of $B$ to $H_{0}$. Formula (3) holds true if instead of $B$ and $U_{t}, t>0$, we apply it to their restrictions to $H_{0}$.

For the classes of operators under consideration, the spectral type of an operator is defined as the class of all operators that are unitarily equivalent to the original one. Since unitary groups and isometric semigroups are uniquely determined by their cogenerators and vice versa, it is natural to define the spectral type of a group (semigroup) via the spectral type of its cogenerator. For the class of unitary operators, the spectral type is determined by a scalar measure on the unit circle $\mathbb{T}$ with respect to which the spectral measure of a unitary operator is absolutely continuous, and an integer-valued function on $\mathbb{T}$ counting the local multiplicity of the unitary operator at almost all points relative to the measure. In accordance with the Wold-Kolmogorov decomposition of the cogenerator, every strongly continuous isometric semigroup splits into a direct sum of its unitary and pure isometric parts. The former is a semigroup of unitary operators indexed by $\mathbb{R}_{+}$, and it extends naturally to a unitary group with indices in $\mathbb{R}$. The latter is a semigroup of completely nonunitary operators, i.e., operators unitarily equivalent to a unilateral shift. Thus, the spectral type of an isometric semigroup is determined by the spectral type of the cogenerator of its unitary part and by the multiplicity of the unilateral shifts.

The following (probably, known) statement shows that the multiplicity of the unilateral shift cannot be changed by compact perturbations of the semigroup, while trace class perturbations preserve the absolutely continuous part of the cogenerator. To make 
the exposition self-contained, we present a short proof of this fact. The proof below was communicated to the authors by R. V. Romanov.

Theorem 2.1. Let $\left(V_{t}\right)_{t \geq 0}$ and $\left(\tilde{V}_{t}\right)_{t \geq 0}$ be strongly continuous semigroups of isometric operators in a Hilbert space $H$.

1) If the operator $\widetilde{V}_{t}-V_{t}$ is compact for all $t \geq 0$, then the pure isometric parts of the semigroups $\left(V_{t}\right)_{t \geq 0}$ and $\left(\tilde{V}_{t}\right)_{t \geq 0}$ are unitarily equivalent, i.e., the multiplicities of the shifts coincide.

2) If $\widetilde{V}_{t}-V_{t} \in \mathfrak{S}_{1}$ for all $t \geq 0$, then the absolutely continuous parts of the semigroups $\left(V_{t}\right)_{t \geq 0}$ and $\left(\widetilde{V}_{t}\right)_{t \geq 0}$ are unitarily equivalent.

For the proof of the theorem we need the following lemma.

Lemma 2.2. Let $T(t), t \geq 0$, be a strongly continuous family of compact operators such that $\sup _{t \geq 0}\|T(t)\|<\infty$. Then the operator $\int_{0}^{\infty} e^{-t} T(t) d t$ is compact.

Proof. For any $t$, consider the Schmidt expansion $T(t)=\sum_{j=1}^{\infty} s_{t j}\left(\cdot, x_{t j}\right) y_{t j}$, where $\left(x_{t j}\right)$, $\left(y_{t j}\right)$ are orthonormal systems and $s_{t j} \searrow 0$. Note that the functions $t \mapsto s_{t j}$ are continuous from below for all $j$, and therefore they are measurable. The functions $t \mapsto x_{t j}$ and $t \mapsto y_{t j}$ are also measurable. The norm of the operator $\sum_{j \geq n} s_{t j}\left(h, x_{t j}\right) y_{t j}$ equals $s_{t n}$, whence

$$
\left\|\int_{0}^{\infty} e^{-t} \sum_{j \geq n} s_{t j}\left(\cdot, x_{t j}\right) y_{t j} d t\right\| \leq \int_{0}^{\infty} e^{-t} s_{t n} d t
$$

By hypothesis, $\sup _{t \geq 0} s_{t n} \leq \sup _{t \geq 0}\|T(t)\|<\infty$. Since for any $t$ we have $s_{t n} \rightarrow 0$ as $n \rightarrow \infty$, the Lebesgue theorem yields $\int_{0}^{\infty} e^{-t} s_{t n} d t \rightarrow 0$, so that

$$
\left\|\int_{0}^{\infty} e^{-t} \sum_{j \geq n} s_{t j}\left(\cdot, x_{t j}\right) y_{t j} d t\right\| \rightarrow 0
$$

as $n \rightarrow \infty$.

It remains to show that the operator $\int_{0}^{\infty} e^{-t} \sum_{j<n} s_{t j}\left(\cdot, x_{t j}\right) y_{t j} d t$ is compact for all $n$. For this, note that the norms of the operators $\left(\cdot, x_{t j}\right) y_{t j}$ in any class $\mathfrak{S}_{p}$ are equal to 1 , whence the $\mathfrak{S}_{p}$-norms of the operators $\int_{0}^{\infty} e^{-t} \sum_{j=1}^{n-1} s_{t j}\left(\cdot, x_{t j}\right) y_{t j} d t$ do not exceed

$$
\int_{0}^{\infty} e^{-t} \sum_{j=1}^{n-1} s_{t j} d t \leq(n-1) \cdot \sup _{t \geq 0}\|T(t)\| \int_{0}^{\infty} e^{-t} d t=(n-1) \cdot \sup _{t \geq 0}\|T(t)\| .
$$

Proof of Theorem 2.1. We prove statement 1) of the theorem. Applying Lemma 2.2 to $T(t)=\widetilde{V}_{t}-V_{t}$, we see that the operator $\int_{0}^{\infty} e^{-t}\left(\tilde{V}_{t}-V_{t}\right) d t$ is compact. This means that the difference of the cogenerators of the semigroups $\left(V_{t}\right)$ and $\left(\widetilde{V}_{t}\right)$ is compact. Hence, the cogenerators have equal Fredholm indices, which are exactly the multiplicities of the shift.

Now we prove statement 2). Denote by $Q$ the subspace where the cogenerator of the semigroup $\left(\tilde{V}_{t}\right)$ acts as an absolutely continuous unitary operator. Denote by $Z$ the natural embedding of $Q$ into $H$. The assumption $\widetilde{V}_{t}-V_{t} \in \mathfrak{S}_{1}$ implies that $Z\left(\left.\tilde{V}_{t}\right|_{Q}\right)-\gamma_{t} Z \in \mathfrak{S}_{1}$, where $\left(\gamma_{t}\right)_{t \in \mathbb{R}}$ is the unitary dilation of the semigroup $\left(V_{t}\right)$. Then, by the classical scattering theory for a pair of unitary operators, a strong limit of the isometries $\gamma_{t} Z\left(\left.\widetilde{V}_{t}\right|_{Q}\right)^{-1}$ exists as $t \rightarrow+\infty$, which gives rise to an isometric wave operator $W$ (see [12, Theorem 6.5.5]). By construction, the range of the operator $W$ is contained in $H$ and reduces the group $\left(\gamma_{t}\right)$. Hence, the operator $W$ realizes a unitary equivalence between the restriction of the semigroup $\left(\widetilde{V}_{t}\right)$ to $Q$ and some unitary part of the semigroup $\left(V_{t}\right)$. 
We have shown that the absolutely continuous unitary part of the semigroup $\left(\tilde{V}_{t}\right)$ is unitarily equivalent to some part of the semigroup $\left(V_{t}\right)$. Similarly, the absolutely continuous unitary part of the semigroup $\left(V_{t}\right)$ is unitarily equivalent to some part of the semigroup $\left(\tilde{V}_{t}\right)$. Then, by the spectral theorem, the absolutely continuous unitary parts of the semigroups $\left(V_{t}\right)$ and $\left(\tilde{V}_{t}\right)$ are unitarily equivalent.

We have an immediate consequence for the perturbations of the semigroup of shifts.

Corollary 2.3. Let $\left(\tau_{t}\right)_{t \geq 0}$ be the semigroup of shifts, and let $\left(\widetilde{\tau}_{t}\right)_{t \geq 0}$ be a strongly continuous semigroup of isometric operators in $L^{2}\left(\mathbb{R}_{+}\right)$.

1) If the operator $\widetilde{\tau}_{t}-\tau_{t}$ is compact for all $t \geq 0$, then there exists a unitary group $\left(\omega_{t}\right)_{t \in \mathbb{R}}$ such that the semigroup $\left(\widetilde{\tau}_{t}\right)$ is unitarily equivalent to the direct sum $\left(\tau_{t}\right) \oplus\left(\omega_{t}\right)$, $t \geq 0$.

2) If, moreover, $\widetilde{\tau}_{t}-\tau_{t} \in \mathfrak{S}_{1}$ for $t \geq 0$, then the spectral measure (of each element, or equivalently, of the cogenerator) for the group $\left(\omega_{t}\right)$ is singular.

For the model on the unit circle, Corollary 2.3 means that under the assumption $\phi_{t}(\widetilde{S})-\phi_{t}(S) \in \mathfrak{S}_{1}, t>0$, the multiplicity of the shift for the operator $\widetilde{S}$ is 1 , and the spectral measure of its unitary part is necessarily singular with respect to Lebesgue measure.

\section{§3. The spaces $K_{\theta}$ And the Model COnstruction}

In this section, starting with a singular measure $\mu$ on the unit circle $\mathbb{T}$ and an inner function $\theta$ associated with $\mu$, we introduce a special model of a perturbation satisfying (i)-(iii).

Let $\mu$ be a measure on $\mathbb{T}$ that is singular relative to the Lebesgue measure $m$, and let $\mu(\{1\})=0$. Define the function $\theta$ by

$$
\frac{1+\theta(z)}{1-\theta(z)}=\int_{\mathbb{T}} \frac{1+\bar{\xi} z}{1-\bar{\xi} z} d \mu(\xi), \quad z \in \mathbb{D} .
$$

It is well known that $\theta$ is an inner function. The measure $\mu$ is supported on the set where the angular boundary limits of $\theta$ exist and are equal to 1 . The measure $\mu$ is said to be a Clark measure of the function $\theta$.

For $u \in L^{2}(\mu)$, put

$$
(\Omega u)(z)=(1-\theta(z)) \int_{\mathbb{T}} \frac{u(\xi) d \mu(\xi)}{1-\bar{\xi} z} .
$$

Clark [18] proved that $\Omega$ is a unitary operator from $L^{2}(\mu)$ to $K_{\theta}$. Moreover, the angular boundary values of the function $\Omega u$ exist and coincide with $u \quad \mu$-almost everywhere [9]. Analogs of these results for the spaces of vector-valued functions can be found in 20 .

In this section, $V$ is the operator of multiplication by the independent variable $\xi$ on $L^{2}(\mu)$. Note that 1 is not an eigenvalue for $V$, because $\mu(\{1\})=0$. We find a formula for the unitary operator $\Omega V \Omega^{*}$, which is a unitarily equivalent transplantation of $V$ to $K_{\theta}$. For $h=\Omega u, u \in L^{2}(\mu)$, we have

$$
\begin{aligned}
\left(\Omega V \Omega^{*} h\right)(z)-z h(z) & =(\Omega V u)(z)-z(\Omega u)(z) \\
& =(1-\theta(z)) \int_{\mathbb{T}} \frac{(\xi-z) u(\xi) d \mu(\xi)}{1-\bar{\xi} z}=(1-\theta(z)) \int_{\mathbb{T}} \xi u(\xi) d \mu(\xi) .
\end{aligned}
$$

Since $\int_{\mathbb{T}} \xi u(\xi) d \mu(\xi)=(u, \bar{\xi})_{L^{2}(\mu)}=(h, \Omega \bar{\xi})_{K_{\theta}}$, we obtain

$$
\Omega V \Omega^{*} h=z h+(h, g)(1-\theta), \quad h \in K_{\theta},
$$


where $g=\Omega \bar{\xi} \in K_{\theta}$ (it is easy to check the formula for $g, g(z)=\frac{\theta(z)-\theta(0)}{z(1-\theta(0))}$, which will not be used in this paper).

Recall that $S$ denotes the shift operator on $H^{2}$; we define the operator $\widetilde{S}$ on $H^{2}$ by

$$
\widetilde{S}=S+(\cdot, g)(1-\theta) .
$$

We have shown that $K_{\theta}$ is an invariant subspace for $\widetilde{S}$, and that the restriction of $\widetilde{S}$ to $K_{\theta}$ is unitarily equivalent to $V$. Clearly, $\widetilde{S}$ coincides with $S$ on $K_{\theta}$. We arrive at the following statement.

Proposition 3.1. For the operator $\widetilde{S}$ defined by ([6), properties (i)-(iii) are fulfilled; the restriction of $\widetilde{S}$ to $K_{\theta}$ is unitarily equivalent to the operator $V$ of multiplication by the independent variable on the space $L^{2}(\mu)$. Thus, $\widetilde{S}$ is unitarily equivalent to $S \oplus V$.

The operator $\widetilde{S}$ differs from the multiplication by $z$ by a rank-one operator: $\widetilde{S}-S=$ $(\cdot, g)(1-\theta)$, the norm of which satisfies

$$
\|\widetilde{S}-S\|=\|g\|_{K_{\theta}} \cdot\|1-\theta\|_{H^{2}}=\|\bar{\xi}\|_{L^{2}(\mu)} \cdot\|1-\theta\|_{H^{2}}<2 \sqrt{\mu(\mathbb{T})} .
$$

We shall work mainly with measures $\mu$ satisfying the additional condition

$$
\int_{\mathbb{T}} \frac{d \mu(\xi)}{|1-\xi|^{2}}<\infty .
$$

This implies, in particular, that $\mu(\{1\})=0$. Condition (8) is well known in the theory of inner functions and model subspaces. It is equivalent to any of the following (see, e.g., 22, Chapter VI]):

(i) the function $\theta$ defined by (41) has a finite angular derivative at the point 1 ;

(ii) each function in $K_{\theta}$ has a finite nontangential limit at the point 1.

Moreover, the function $\frac{1-\overline{\theta(1)} \theta}{1-z}$ belongs to $K_{\theta}$ and is the reproducing kernel at the point 1 ,

$$
\left\|\frac{1-\overline{\theta(1)} \theta}{1-z}\right\|_{K_{\theta}}^{2}=\left\|\frac{1-\overline{\theta(1)}}{1-z}\right\|_{L^{2}(\mu)}^{2}<4 \int_{\mathbb{T}} \frac{d \mu(\xi)}{|1-\xi|^{2}} .
$$

\section{§4. Equivalent MOdels}

Our initial construction was a model of shifts in the space $L^{2}\left(\mathbb{R}_{+}\right)$. We shall also work with other models, which are unitarily equivalent to the original one.

An equivalent model construction on the real line can be obtained from the model of shifts via the Fourier transform. It sends the space $L^{2}\left(\mathbb{R}_{+}\right)$to the Hardy space $H^{2}\left(\mathbb{C}_{+}\right)$ in the upper half-plane. The isometric semigroup $\left(\tau_{t}\right)_{t \geq 0}$ of shifts becomes the semigroup of operators of multiplication by the functions $\exp (i t z)$.

In the case of the model on the unit circle $\mathbb{T}$, we work in the Hardy space $H^{2}$, which is a subspace of $L^{2}$. In the notation $L^{2}$ and $H^{2}$ we omit the measure, meaning the normalized Lebesgue measure $m$ on $\mathbb{T}$ such that $m(\mathbb{T})=1$. The cogenerator of the unperturbed isometric semigroup is the operator $S$ of multiplication by the independent variable $z$. Then the semigroup consists of operators of the form $\phi_{t}(S)$ with $\phi$ defined by (2). In other words, our isometric semigroup is the group of operators of multiplication by the inner functions $\phi_{t}, t \geq 0$.

Now we list some formulas establishing a unitary equivalence of the models on the unit circle $\mathbb{T}$ and on the real line $\mathbb{R}$. For the variable $z$ on $\mathbb{T}$ we write $x=i \frac{1+z}{1-z} \in \mathbb{R}$. Given a measure $\mu$ on $\mathbb{T}$, define the measure $\nu$ on $\mathbb{R}$ by

$$
d \mu(z)=\frac{d \nu(x)}{\pi\left(1+x^{2}\right)} .
$$


Condition (8) is equivalent to $\nu(\mathbb{R})<\infty$. The mapping

$$
u \mapsto v, \quad v(x)=\frac{1}{\sqrt{\pi}(x+i)} \cdot u\left(\frac{x-i}{x+i}\right),
$$

is a unitary operator from $L^{2}(\mu)$ onto $L^{2}(\nu)$, and also from $L^{2}=L^{2}(\mathbb{T})$ to $L^{2}(\mathbb{R})$; in the latter case the Hardy class $H^{2}$ is taken onto $H^{2}\left(\mathbb{C}_{+}\right)$. The function $u \in L^{2}(\mu)$ is expressed via $v \in L^{2}(\nu)$ as

$$
u(z)=\frac{2 i \sqrt{\pi}}{1-z} \cdot v\left(i \frac{1+z}{1-z}\right) .
$$

\section{§5. Functions of the Operators $S$ And $\widetilde{S}$}

Now we deal with the construction where $\widetilde{S}$ is a rank-one perturbation of $S$ defined by (66). Take a function $\phi \in H^{\infty}$ and suppose that the values of $\phi$ are defined $\mu$-almost everywhere.

On the subspace $\theta H^{2}$, the operator $\widetilde{S}$ coincides with $S$, so that $\phi(\widetilde{S})$ coincides with $\phi(S)$ there. Thus, we need to study only the restriction of $\phi(\widetilde{S})-\phi(S)$ to $K_{\theta}$, which makes it natural to consider the operator

$$
X: L^{2}(\mu) \rightarrow H^{2}, \quad X=(\phi(\widetilde{S})-\phi(S)) \Omega,
$$

with $\Omega$ defined by (5). Take $u \in L^{2}(\mu)$. Then

$$
\begin{aligned}
(X u)(z) & =(1-\theta(z)) \int_{\mathbb{T}} \frac{\phi(\xi) u(\xi)}{1-\bar{\xi} z} d \mu(\xi)-\phi(z)(1-\theta(z)) \int_{\mathbb{T}} \frac{u(\xi)}{1-\bar{\xi} z} d \mu(\xi) \\
& =(1-\theta(z)) \int_{\mathbb{T}} \frac{\phi(\xi)-\phi(z)}{\xi-z} \xi u(\xi) d \mu(\xi), \quad u \in L^{2}(\mu) .
\end{aligned}
$$

Since $\Omega$ is a unitary operator from $L^{2}(\mu)$ onto $K_{\theta}$, the operator $\phi(\widetilde{S})-\phi(S)$ belongs to $\mathfrak{S}_{p}$ if and only if the operator $X$ belongs to $\mathfrak{S}_{p}$, and the $\mathfrak{S}_{p}$-norms of these operators coincide.

Relation (10) establishes unitary correspondences between $L^{2}(\mu)$ and $L^{2}(\nu)$, and between $H^{2}$ and $H^{2}\left(\mathbb{C}_{+}\right)$. Using these identifications, we construct the operator

$$
Y: L^{2}(\nu) \rightarrow H^{2}\left(\mathbb{C}_{+}\right)
$$

as a unitary transplantation of $X$. We have

$$
\begin{aligned}
(Y v)(x) & =\frac{1}{\sqrt{\pi}(x+i)}(X u)\left(\frac{x-i}{x+i}\right) \\
& =\frac{1}{\sqrt{\pi}(x+i)} \cdot(1-\theta(z)) \int_{\mathbb{T}} \frac{\phi(\xi)-\phi(z)}{\xi-z} \xi u(\xi) d \mu(\xi) \\
& =(1-\Theta(x)) \cdot \frac{1}{2 \pi i} \int_{\mathbb{R}} \frac{\psi(\zeta)-\psi(x)}{\zeta-x} v(\zeta) d \nu(\zeta), \quad v \in L^{2}(\nu),
\end{aligned}
$$

where

$$
\psi(x)=\phi\left(\frac{x-i}{x+i}\right), \quad \Theta(x)=\theta\left(\frac{x-i}{x+i}\right), \quad x \in \mathbb{C}_{+} .
$$

Note that if $\theta$ is defined by (4), then for $\Theta$ we have the formula

$$
\frac{1+\Theta(x)}{1-\Theta(x)}=\frac{1}{\pi i} \int\left(\frac{1}{\zeta-x}-\frac{\zeta}{1+\zeta^{2}}\right) d \nu(\zeta), \quad x \in \mathbb{C}_{+} .
$$

The following proposition follows directly from the construction of $Y$.

Proposition 5.1. The operators $\phi(\widetilde{S})-\phi(S)$ and $Y$ belong or do not belong to the ideals $\mathfrak{S}_{p}$ simultaneously, and $\|\phi(\widetilde{S})-\phi(S)\|_{\mathfrak{S}_{p}}=\|Y\|_{\mathfrak{S}_{p}}$. 


\section{§6. Estimates FOR The Hilbert-Schmidt Class}

Define the integral operator $K: L^{2}(\nu) \rightarrow H^{2}\left(\mathbb{C}_{+}\right)$by

$$
(K v)(x)=\frac{1}{2 \pi i} \int \frac{\psi(\zeta)-\psi(x)}{\zeta-x} v(\zeta) d \nu(\zeta), \quad v \in L^{2}(\nu)
$$

If $K \in \mathfrak{S}_{p}$, then $Y \in \mathfrak{S}_{p}$ as well, and we obviously have

$$
\|Y\|_{\mathfrak{S}_{p}} \leq 2 \cdot\|K\|_{\mathfrak{S}_{p}}
$$

For the norm of $K$ in $\mathfrak{S}_{2}$ we obtain

$$
\|K\|_{\mathfrak{S}_{2}}^{2}=\frac{1}{4 \pi^{2}} \iint_{\mathbb{R} \times \mathbb{R}}\left|\frac{\psi(\zeta)-\psi(x)}{\zeta-x}\right|^{2} d x d \nu(\zeta) .
$$

Consider the semigroups of the form $\left(\phi_{t}(S)\right),\left(\phi_{t}(\widetilde{S})\right)$, where $\phi_{t}(z)=\exp \left(t \frac{z+1}{z-1}\right), t>0$. The corresponding functions $\psi_{t}$ have the form

$$
\psi_{t}(x)=\phi_{t}\left(\frac{x-i}{x+i}\right)=e^{i t x}
$$

Now we state a condition on $\nu$ ensuring that the operator $K$ belongs to the HilbertSchmidt class for all $t$ at once. To this end, we shall obtain a precise formula for the integral in (16) with $\psi=\psi_{t}$; it will give us a sufficient condition for $\phi_{t}(\widetilde{S})-\phi_{t}(S) \in \mathfrak{S}_{2}$ for all $t$. At the end of the section we present an example of the construction for which $\phi_{t}(\widetilde{S})-\phi_{t}(S)$ is not in the Hilbert-Schmidt class for any $t \neq 0$.

Proposition 6.1. Assume that (8) is fulfilled. Then for any $t>0$ we have

$$
\iint_{\mathbb{R} \times \mathbb{R}}\left|\frac{\psi_{t}(\zeta)-\psi_{t}(x)}{\zeta-x}\right|^{2} d x d \nu(\zeta)=2 \pi t \cdot \nu(\mathbb{R})=8 \pi^{2} t \int_{\mathbb{T}} \frac{d \mu(\xi)}{|1-\xi|^{2}}<\infty
$$

Proof. For the integral with respect to Lebesgue measure on the left-hand side, we obtain

$$
\int_{\mathbb{R}} \frac{\left|e^{i t \zeta}-e^{i t x}\right|^{2}}{(\zeta-x)^{2}} d x=4 \int_{\mathbb{R}} \frac{\sin ^{2} \frac{t}{2}(\zeta-x)}{(\zeta-x)^{2}} d x=2 \pi t
$$

where we have used the well-known formula $\int_{\mathbb{R}} \frac{\sin ^{2} \alpha s}{s^{2}} d s=\pi|\alpha|$. The right identity in (17) follows from the relation $\nu(\mathbb{R})=4 \pi \int \frac{d \mu(\xi)}{|1-\xi|^{2}}$.

Corollary 6.2. If $\mu$ satisfies (죠), then $\phi_{t}(\widetilde{S})-\phi_{t}(S) \in \mathfrak{S}_{2}$ for all $t>0$;

$$
\left\|\phi_{t}(\widetilde{S})-\phi_{t}(S)\right\|_{\mathfrak{S}_{2}} \leq 2 \sqrt{2 t}\left(\int_{\mathbb{T}} \frac{d \mu(\xi)}{|1-\xi|^{2}}\right)^{1 / 2} .
$$

Proof. This follows directly from Proposition 5.1 and relations (15), (16), and (17).

In $\S 7$ we find a certain "smallness" condition for a measure $\mu$ at the point 1, see (21), which is sufficient for the inclusion of the operator $\phi_{t}(\widetilde{S})-\phi_{t}(S)$ into the trace class $\mathfrak{S}_{1}$.

The representation (13) of $Y$ in the form of an integral operator implies that $Y \in \mathfrak{S}_{2}$ if and only if

$$
\iint_{\mathbb{R} \times \mathbb{R}}|1-\Theta(z)|^{2}\left|\frac{\psi(\zeta)-\psi(x)}{\zeta-x}\right|^{2} d x d \nu(\zeta)<\infty .
$$

We expect that the condition $\nu(\mathbb{R})<\infty$, equivalent to (8), is also necessary for the inclusions $\phi_{t}(\widetilde{S})-\phi_{t}(S) \in \mathfrak{S}_{2}$, that is, the factor $1-\Theta(z)$ does not essentially affect the convergence of the integral in (16).

Now we present an example of a measure $\nu$ such that the corresponding operator $\phi_{t}(\widetilde{S})-\phi_{t}(S)$ is not in the Hilbert-Schmidt class for any $t \neq 0$. Recall that $\widetilde{S}-S$ is a rank-one operator whose norm can be made arbitrarily small. 
Example 6.3. Let $\nu=\sum_{n \in \mathbb{Z}} \delta_{n}$ be the sum of unit point masses at all integers. Thus, the total mass of $\nu$ is infinite, and hence the corresponding measure $\mu$ on $\mathbb{T}$ does not satisfy condition (8). Putting $\psi_{t}(x)=e^{i t x}$ in the last formula, we obtain the integral

$$
\int_{\mathbb{R}}|1-\Theta(s)|^{2}\left(\sum_{n \in \mathbb{Z}} \frac{\sin ^{2} \frac{t}{2}(s-n)}{(s-n)^{2}}\right) .
$$

Obviously, for any $t \neq 0$, there exist positive constants $\delta(t)<1 / 2$ and $C(t)$ such that the sum in parentheses in the above formula is bounded below by $C(t)$ for $s \in(k+$ $\delta(t), k+2 \delta(t)), k \in \mathbb{Z}$. On the other hand, an elementary estimate shows that for $s \in(k+\delta(t), k+2 \delta(t)), k \in \mathbb{Z}$, we have

$$
\left|\frac{1+\Theta(s)}{1-\Theta(s)}\right|=\left|\sum_{n \in \mathbb{Z}} \frac{1+s n}{(s-n)\left(n^{2}+1\right)}\right| \leq C_{1}(t),
$$

whence $|1-\Theta(s)| \geq C_{2}(t)>0$. Combining these estimates, we conclude that the integral diverges, i.e., $Y$ does not belong to the Hilbert-Schmidt class.

\section{$\S 7$. Estimates FOR THE TRACE CLASS}

In this section, instead of the Hilbert-Schmidt class $\mathfrak{S}_{2}$, we consider the classes $\mathfrak{S}_{p}$ with $p<2$, and first of all, the trace class $\mathfrak{S}_{1}$. In accordance with (15), to show that $\phi_{t}(\widetilde{S})-\phi_{t}(S) \in \mathfrak{S}_{p}$, we need to prove that $K \in \mathfrak{S}_{p}$, where $K$ is defined by (14) with $\psi=\psi_{t}, \psi_{t}(x)=e^{i t x}$. We reduce this problem to a question on embeddings of the PaleyWiener space $\mathcal{P} \mathcal{W}_{t}$ of all the entire functions of exponential type at most $t$ that are square integrable on $\mathbb{R}$. Embedding operators for spaces of analytic functions and their inclusion to ideals $\mathfrak{S}_{p}$ were studied in detail by O. G. Parfenov [7, 8]. In [3], generalizations of some of Parfenov's results to embeddings of coinvariant subspaces $K_{\theta}$ were obtained.

Consider the adjoint to the operator $K$ defined by (14) with $\psi=\psi_{t}, \psi_{t}(x)=e^{i t x}$ :

$$
\begin{aligned}
\left(K^{*} f\right)(\zeta) & =-\frac{1}{2 \pi i} \int \frac{e^{-i t \zeta}-e^{-i t x}}{\zeta-x} f(x) d x \\
& =e^{-\frac{i t \zeta}{2}} \int e^{-\frac{i t x}{2}} f(x)\left(\frac{1}{\pi} \cdot \frac{\sin \frac{t}{2}(x-\zeta)}{x-\zeta}\right) d x, \quad f \in L^{2}(\mathbb{R}) .
\end{aligned}
$$

It is well known that the function $\frac{1}{\pi} \cdot \frac{\sin \frac{t}{2}(x-\zeta)}{x-\zeta}$ is the reproducing kernel at $\zeta$ for the space $\mathcal{P} \mathcal{W}_{t / 2}$, which means that this function belongs to $\mathcal{P} \mathcal{W}_{t / 2}$ and the inner product of any $f \in \mathcal{P} \mathcal{W}_{t / 2}$ with the reproducing kernel equals $f(\zeta)$. Therefore, $K^{*} f=0$ if the function $\tilde{f}, \tilde{f}(x)=e^{-\frac{i t x}{2}} f(x)$, is orthogonal to $\mathcal{P} \mathcal{W}_{t / 2}$, and if $\tilde{f} \in \mathcal{P} \mathcal{W}_{t / 2}$, then

$$
\left(K^{*} f\right)(\zeta)=e^{-\frac{i t \zeta}{2}} \cdot \tilde{f}(\zeta)=e^{-i t \zeta} f(\zeta)
$$

Thus, $K^{*}$ belongs to $\mathfrak{S}_{p}$ simultaneously with the operator $E_{\nu, t / 2}$ that embeds the space $\mathcal{P} \mathcal{W}_{t / 2}$ in $L^{2}(\nu)$.

The following criterion is due to Parfenov [7, 8].

Theorem 7.1. Let $\Delta_{n}=[n, n+1)$. Then $E_{\nu, t} \in \mathfrak{S}_{p}, p>0$, if and only if

$$
\sum_{n \in \mathbb{Z}}\left(\nu\left(\Delta_{n}\right)\right)^{p / 2}<\infty \text {. }
$$

Moreover,

$$
\left\|E_{\nu, t}\right\|_{\mathfrak{S}_{p}}^{p} \leq C(p) t^{p / 2} \sum_{n \in \mathbb{Z}}\left(\nu\left(\Delta_{n}\right)\right)^{p / 2} .
$$


Going back to the measure $\mu$, we see that condition (19) reads

$$
\sum_{n \in \mathbb{Z}}\left(\int_{\gamma_{n}} \frac{d \mu(\xi)}{|1-\xi|^{2}}\right)^{p / 2}<\infty
$$

where the $\operatorname{arcs} \gamma_{n}$ are defined for $n>0$ by $\gamma_{n}=\left\{e^{i \phi}: \pi /(n+1) \leq \phi \leq \pi / n\right\}$ and symmetrically for $n<0$.

We obtain a condition that is similar to (8) and is sufficient for (19) and, therefore, is sufficient for the inclusion of $\phi_{t}(\widetilde{S})-\phi_{t}(S)$ into $\mathfrak{S}_{p}$.

Proposition 7.2. Let $0<p<2$. If the measure $\mu$ satisfies

$$
\int_{\mathbb{T}} \frac{d \mu(\xi)}{|1-\xi|^{q}}<\infty
$$

for some $q>1+2 / p$, then $K \in \mathfrak{S}_{p}$, and consequently $\phi_{t}(\widetilde{S})-\phi_{t}(S) \in \mathfrak{S}_{p}$.

In particular, (21) with $q>3$ yields $\phi_{t}(\widetilde{S})-\phi_{t}(S) \in \mathfrak{S}_{1}$, and

$$
\left\|\phi_{t}(\widetilde{S})-\phi_{t}(S)\right\|_{\mathfrak{S}_{1}} \leq M_{q} \cdot t^{1 / 2} \cdot\left(\int_{\mathbb{T}} \frac{d \mu(\xi)}{|1-\xi|^{q}}\right)^{1 / 2}
$$

where $M_{q}$ is a constant depending only on $q$.

Proof. We rewrite condition (21) in terms of the measure $\nu$ defined by (9):

$$
\int_{\mathbb{R}}\left(x^{2}+1\right)^{r / 2} d \nu(x)=2^{-q} \pi \int_{\mathbb{T}} \frac{d \mu(\xi)}{|1-\xi|^{q}}<\infty,
$$

where $r=q-2>(2-p) / p$. Then, by the Hölder inequality with exponents $2 / p$ and $2 /(2-p)$,

$$
\begin{aligned}
\left(\sum_{n \in \mathbb{Z}}\left(\nu\left(\Delta_{n}\right)\right)^{p / 2}\right)^{2 / p} & \leq \sum_{n \in \mathbb{Z}}(|n|+1)^{r} \nu\left(\Delta_{n}\right) \cdot\left(\sum_{n \in \mathbb{Z}}(|n|+1)^{-p r /(2-p)}\right)^{(2-p) / p} \\
& =\text { const } \cdot \sum_{n \in \mathbb{Z}}(|n|+1)^{r} \nu\left(\Delta_{n}\right) \leq \text { const } \cdot \int_{\mathbb{R}}(|t|+1)^{r} d \nu(t) .
\end{aligned}
$$

Now the claim follows from Parfenov's theorem and inequality (20).

Example 7.3. The exponent 3 is sharp, and for $q=3$ the second statement of Proposition 7.2 fails. Indeed, choose $\nu$ so that $\nu\left(\Delta_{n}\right)=((|n|+1) \log (|n|+2))^{-2}$. Then (23) is fulfilled, but (19) is not, so that $K$ is not in $\mathfrak{S}_{1}$.

\section{$\S 8$. Operators of MULTiplicity GREATER THAN 1}

Now we use the construction developed above in the case of multiplicity 1 , for a generalization to operators with arbitrary multiplicity.

Let $\left\{\mu_{n}\right\}$ be a family of singular measures on the unit circle, where $n$ runs over the set $n=1,2, \ldots, N$ for some positive integer $N$ or over $\mathbb{N}$. We assume that

$$
\sum_{n}\left(\int_{\mathbb{T}} \frac{d \mu_{n}(\xi)}{|1-\xi|^{q}}\right)^{1 / 2}<\infty
$$

for some $q>3$. 
For each $n$, we construct objects as in $\S 3$ : the function $\theta_{n}$ is determined by formula (4) with $\mu_{n}$ in place of $\mu$, the operator $\Omega_{n}: L^{2}\left(\mu_{n}\right) \rightarrow K_{\theta_{n}}$ acts by formula (5) with $g_{n}=\Omega_{n} \bar{\xi}$, and $V_{n}$ is the operator of multiplication by $z$ on $L^{2}\left(\mu_{n}\right)$. Set

$$
\widehat{\theta}_{n}=\prod_{k=1}^{n-1} \theta_{k} .
$$

The operator $\Omega: \sum \oplus L^{2}\left(\mu_{n}\right) \rightarrow H^{2}$ will be defined here by

$$
\Omega\left(\sum \oplus u_{n}\right)=\sum \widehat{\theta}_{n} \Omega_{n} u_{n}
$$

Since condition (24) holds for some $q>3$, it holds for $q=2$. Hence,

$$
\sum_{n}\left\|\frac{1-\overline{\theta_{n}(1)} \theta_{n}}{1-z}\right\|_{L^{2}}<2 \sum_{n}\left(\int_{\mathbb{T}} \frac{d \mu_{n}(\xi)}{|1-\xi|^{2}}\right)^{1 / 2}<\infty .
$$

Therefore, the series

$$
\sum_{n} \overline{\widehat{\theta}_{n}(1)} \widehat{\theta}_{n} \frac{\left(1-\overline{\theta_{n}(1)} \theta_{n}\right)}{1-z}
$$

converges in the norm of the space $L^{2}$. The partial sums have the form $\frac{1-\overline{\widehat{\theta}_{n}(1)} \widehat{\theta}_{n}}{1-z}$. Hence, the limit function can be written as $\frac{1-\theta}{1-z}$ for an inner function $\theta$ that coincides with the product of the functions $\theta_{n}$ up to a unimodular multiplicative constant. It is easily seen that $\Omega$ maps $\sum \oplus L^{2}\left(\mu_{n}\right)$ onto $K_{\theta}$ isometrically.

Define the operator $\widetilde{S}$ on $H^{2}$ by

$$
\widetilde{S}=S+\sum_{n}\left(\cdot, \widehat{\theta}_{n} g_{n}\right) \widehat{\theta}_{n} \cdot\left(1-\theta_{n}\right),
$$

which is a generalization of (6). Then the operator $\widetilde{S}$ is diagonal with respect to the decomposition $H^{2}=\sum \oplus \widehat{\theta}_{n} K_{\theta_{n}} \oplus \theta H^{2}$. Indeed, $\widetilde{S}$ coincides with $S$ on $\theta H^{2}$, while on each subspace $\widehat{\theta}_{n} K_{\theta_{n}}$ the operator $\widetilde{S}$ is a unitary transplantation of multiplication by $z$ on $L^{2}\left(\mu_{n}\right)$. By a direct computation, we obtain $\widetilde{S}\left(\widehat{\theta}_{n} f\right)=\widehat{\theta}_{n} \Omega_{n}(z u) \in \widehat{\theta}_{n} K_{\theta_{n}}$ for $f=\Omega_{n} u \in K_{\theta_{n}}$.

Thus, $\widetilde{S}$ satisfies properties (i)-(iii). Much as in the case of (17), we obtain an estimate for the trace class norm of $\widetilde{S}-S$ :

$$
\|\widetilde{S}-S\|_{\mathfrak{S}_{1}}<2 \sum_{n} \sqrt{\mu_{n}(\mathbb{T})} .
$$

To estimate the trace class norm of $\phi_{t}(\widetilde{S})-\phi_{t}(S)$, we use inequality (22), which gives us

$$
\left\|\phi_{t}(\widetilde{S})-\phi_{t}(S)\right\|_{\mathfrak{S}_{1}} \leq M_{q} \cdot t^{1 / 2} \cdot \sum_{n}\left(\int_{\mathbb{T}} \frac{d \mu_{n}(\xi)}{|1-\xi|^{q}}\right)^{1 / 2} .
$$

Proof of Theorem 1.1. Take an arbitrary unitary operator $V$ whose spectral measure is singular relative to Lebesgue measure and has no point mass at 1 . Then there exist singular unitary operators $V_{n}$ of multiplicity 1 such that $V=\sum \oplus V_{n}$. We construct measures $\mu_{n}$ such that each operator $V_{n}$ is unitarily equivalent to multiplication by the independent variable $\xi$ on $L^{2}\left(\mu_{n}\right)$. We may assume that the measures $\mu_{n}$ satisfy (24) with $q>3$ and $\sum_{n} \sqrt{\mu_{n}(\mathbb{T})}<\varepsilon / 2$; otherwise, these properties can be gained after multiplying the measures $\mu_{n}$ by appropriate positive weights. Consider the operator $\widetilde{S}$ defined by (26). Then (27) yields $\|\widetilde{S}-S\|_{\mathfrak{S}_{1}}<\varepsilon$, and for any $t>0$ the operator $\phi_{t}(\widetilde{S})-\phi_{t}(S)$ is of trace class with norm estimated by (28). 
Remark 8.1. For the Hilbert-Schmidt norm, from (18) we obtain the estimate

$$
\left\|\phi_{t}(\widetilde{S})-\phi_{t}(S)\right\|_{\mathfrak{S}_{2}} \leq 2 \sqrt{2 t}\left(\sum_{n} \int_{\mathbb{T}} \frac{d \mu_{n}(\xi)}{|1-\xi|^{2}}\right)^{1 / 2} .
$$

Proof of Theorem 1.2, Statement 1) is a particular case of Theorem 2.1. Statement 2) follows immediately from Theorem 1.1 when we pass to the model related to the group of shifts on the line. Indeed, in the construction in Theorem 1.1 the differences of the elements of the semigroups on $L^{2}\left(\mathbb{R}_{+}\right)$belong to the trace class, as required.

Proof of Theorem 1.3. Let $V$ be an arbitrary unitary operator such that 1 is not its eigenvalue. Then $V=(A-i I)(A+i I)^{-1}$ for some selfadjoint operator $A$, and $\phi_{t}(V)=$ $\exp (i t A)$. Applying Kuroda's version of the Weil-von Neumann theorem (see 5 and 12, Theorem 6.2.5]) to some cross-normed ideal contained in all ideals $\mathfrak{S}_{p}$ with $p>1$, for a given selfadjoint operator we can construct a close selfadjoint operator with pure point spectrum such that the difference between the perturbed operator and the initial one belongs to $\mathfrak{S}_{p}$ for all $p>1$, and the norms can be taken arbitrarily small.

We represent the operator $A$ as the direct sum of bounded operators $A_{n}$ and, applying the Kuroda theorem, construct operators $A_{n}^{\prime}$ such that the norms $\left\|A_{n}-A_{n}^{\prime}\right\|_{\mathfrak{S}_{p}}$ are small. The results of Davies [19] imply that $\exp \left(i t A_{n}\right)-\exp \left(i t A_{n}^{\prime}\right)$ belongs to all classes $\mathfrak{S}_{p}$, $p>1$, for $0 \leq t \leq 1$. Thus, we can construct a selfadjoint operator $A^{\prime}$ such that $\exp (i t A)-\exp \left(i t A^{\prime}\right)$ is in all classes $\mathfrak{S}_{p}, p>1$, for $0 \leq t \leq 1$. Hence, this holds true for any $t>0$, because the inclusion $\exp (i t A)-\exp \left(i t A^{\prime}\right) \in \mathfrak{S}_{p}$ implies the inclusion $\exp (2 i t A)-\exp \left(2 i t A^{\prime}\right) \in \mathfrak{S}_{p}$. Now the statement follows from Theorem 1.1 applied to the unitary operator $V^{\prime}=\left(A^{\prime}-i I\right)\left(A^{\prime}+i I\right)^{-1}$.

\section{REFERENCES}

[1] G. G. Amosov and A. D. Baranov, On the dilation of contractive cocycles and cocycle perturbations of the translation group on the line, Mat. Zametki 79 (2006), no. 1, 3-18; English transl., Math. Notes 79 (2006), no. 1-2, 3-17. MR.2249142 (2007h:47066)

[2] $ـ$, On the dilation of contractive cocycles and cocycle perturbations of the translation group on the line. II, Mat. Zametki 79 (2006), no. 5, 779-780; English transl., Math. Notes 79 (2006), no. 5-6, 719-720. MR 2249135(2007h:47067)

[3] A. D. Baranov, Embedding of model subspaces of the Hardy class: compactness and Schatten-von Neumann ideals, Izv. Ross. Akad. Nauk Ser. Mat. 73 (2009), no. 6, 3-28; English transl., Izv. Math. 73 (2009), no. 6, 1077-1100. MR2640976

[4] K. Yosida, Functional analysis, Springer-Verlag, Berlin, 1995. MR.1336382 (96a:46001)

[5] T. Kato, Perturbation theory for linear operators, Springer-Verlag, Berlin, 1995. MR.1335452 (96a:47025)

[6] N. K. Nikol'skiü, Lectures on the shift operator, Nauka, Moscow, 1980; English transl., Treatise on the shift operator, Grundlehren Math. Wiss., Bd. 273, Springer-Verlag, Berlin, 1986. MR0575166 (82i:47013) MR0827223 (87i:47042)

[7] O. G. Parfenov, On the properties of embedding operators of some classes of analytic functions, Algebra i Analiz 3 (1991), no. 2, 199-222; English transl., St. Petersburg Math. J. 3 (1992), no. 2, 425-446. MR:1137528 (92k:46032)

[8] - Weighted estimates for the Fourier transform, Zap. Nauchn. Sem. S.-Peterburg. Otdel. Mat. Inst. Steklov. (POMI) 222 (1995), 151-162; English transl., J. Math. Sci. (New York) 87 (1997), no. 5, 3878-3885. MR1359997 (96k:42011)

[9] A. G. Poltoratskiı̌, The boundary behavior of pseudocontinuable functions, Algebra i Analiz 5 (1993), no. 2, 189-210; English transl., St. Petersburg Math. J. 5 (1994), no. 2, 389-406. MR1223178 (94k:30090)

[10] B. Sz.-Nagy and C. Foiaş, Harmonic analysis of operators on Hilbert space, North-Holland Publ. Co., Amsterdam-London, 1970. MR0275190 (43:947)

[11] E. Hille and R. S. Phillips, Functional analysis and semi-groups, Amer. Math. Soc. Colloq. Publ., vol. 31, Amer. Math. Soc., Providence, RI, 1957. MR0089373(19:664d) 
[12] D. R. Yafaev, Mathematical scattering theory. General theory, S.-Peterburg. Univ., St. Petersburg, 1994; English transl., Transl. Math. Monogr., vol. 105, Amer. Math. Soc., Providence, RI, 1992. MR.1180965 (94f:47012) MR1784870 (2001e:47015)

[13] P. R. Ahern and D. N. Clark, Radial limits and invariant subspaces, Amer. J. Math. 92 (1970), 332-342. MR0262511 (41:7117)

[14] _ On functions orthogonal to invariant subspaces, Acta Math. 124 (1970), 191-204. MR 0264385(41:8981a)

[15] G. G. Amosov, Cocycle perturbation of quasifree algebraic K-flow leads to required asymptotic dynamics of associated completely positive semigroup, Infin. Dimens. Anal. Quantum Probab. Relat. Top. 3 (2000), 237-246. MR.1812699 (2001m:46148)

[16] G. G. Amosov and A. D. Baranov, On perturbations of the group of shifts on the line by unitary cocycles, Proc. Amer. Math. Soc. 132 (2004), no. 11, 3269-3273. MR2073301 (2005c:46095)

[17] W. Arveson, Continuous analogues of Fock space, Mem. Amer. Math. Soc. 80 (1989), no. 409, iv+66pp. MR0987590 (90f:47061)

[18] D. N. Clark, One-dimensional perturbations of restricted shifts, J. Anal. Math. 25 (1972), 169-191. MR0301534 (46:692)

[19] E. B. Davies, Lipschitz continuity of functions of operators in the Schatten classes, J. London Math. Soc. (2) 37 (1988), no. 1, 148-157. MR0921753 (89c:47009)

[20] V. Kapustin and A. Poltoratski, Boundary convergence of vector-valued pseudocontinuable functions, J. Funct. Anal. 238 (2006), no. 1, 313-326. MR.2253018 (2007k:47018)

[21] N. K. Nikolski, Operators, functions, and systems: an easy reading. Vols. 1-2, Math. Surveys Monogr., vols. 92-93, Amer. Math. Soc., Providence, RI, 2002. MR.1864396 (2003i:47001a) MR.1892647(2003i:47001b)

[22] D. Sarason, Sub-Hardy Hilbert spaces in the unit disc, Univ. Arkansas Lecture Notes in Math. Sci., vol. 10, Wiley-Intersci., New York, 1994. MR,1289670 (96k:46039)

Moscow Institute of Physics and Technology, Moscow, Russia

E-mail address: gramos@mail.ru

Department of Mathematics and Mechanics, St. Petersburg State University, Stary Petergof, Bibliotechnaya Pl. 2, St. Petersburg 198504, Russia

E-mail address: anton.d.baranov@gmail.com

St. Petersburg Branch, Steklov Mathematical Institute, Russian Academy of Sciences, Fontanka 27, St. Petersburg 191023, Russia

E-mail address: kapustin@pdmi.ras.ru

Received 20/JAN/2010

Translated by THE AUTHORS 